\title{
TECNOLOGIAS DIGITAIS E PRESERVAÇÃO DO PATRIMÔNIO ARQUITETÔNICO: EXPLORANDO ALTERNATIVAS
}

\section{DIGITAL TECHNOLOGIES AND PRESERVATIONOFARCHITECTURAL HERITAGE: EXPLORINGALTERNATIVES}

\section{Cristiane Lopes Canuto ${ }^{1}$}

UFRJ/ FAU/ Programa de Pós Graduação em Arquitetura PROARQ FAU UFRJ, Rio de Janeiro, Rio de Janeiro, Brasil, cricanuto@gmail.com

\section{Larissa Ribeiro Moura ${ }^{2}$}

UFRJ/ FAU/ Programa de Pós Graduação em Arquitetura PROARQ FAU UFRJ, Rio de Janeiro, Rio de Janeiro, Brasil, larissaribeirom@yahoo.com.br

\section{Mônica Santos Salgado ${ }^{3}$}

UFRJ/ FAU/ Programa de Pós Graduação em Arquitetura - PROARQ FAU UFRJ, Rio de Janeiro, Rio de Janeiro, Brasil, monicassalgado@ufrj.br

\section{Resumo}

As tecnologias digitais tem apresentado novas possibilidades em diferentes setores, promovendo alterações no modus operandi em várias áreas do conhecimento. Nesse particular, destacam-se as tecnologias que podem ser utilizadas na aquisição e experimentação de dados que, trabalhados em conjunto, criam um ambiente propício aos arquitetos, auxiliando a tomada de decisão em torno das melhores alternativas de projeto e gestão do processo até a fase de uso-operação e manutenção . Técnicas como o escaneamento a laser, a fotogrametria podem ser trabalhadas concomitantemente com os softwares da Plataforma BIM (Building Information Modelling), gerando modelagens cada vez mais inteligentes e interativas, que permitem a manipulação através das experimentações em Realidade Aumentada(RA) e Realidade Virtual(RV). A preservação digital do patrimônio é uma das áreas do conhecimento a serem beneficiadas com as possibilidades oferecidas pelas novas tecnologias. Dessa forma, este artigo apresenta os resultados da pesquisa cujo objetivo é apresentar vantagens das tecnologias digitais na preservação do patrimônio histórico a partir do levantamento pela captura da realidade com fotogrametria e escaneamento a laser, em integração com BIM , RA e RV. Para isto, realizou-se um estudo de caso com o modelo do painel da fachada do edifício moderno da Faculdade de Arquitetura e Urbanismo na Universidade Federal do Rio de Janeiro- FAU UFRJ. Entende-se a importância da utilização dessas e outras ferramentas na preservação do patrimônio como uma mudança de paradigma, uma vez será possível oferecer a experiência imersiva em edificações de interesse para a preservação, além do registro completo e integração das informações documentais dessas obras.

Palavras-chave: Preservação digital. BIM. Patrimônio Virtual.

\begin{abstract}
The possibilities offered by digital technologies promote changes in the modus operandi from different areas of knowledge. In this regard, we can highlighted the technology to acquire and test data, that can be used together to create an enabling environment for architects, aiding the decision-making around the best design alternatives and process management until the use-operation and maintenance phase. Techniques such as laser scanning and photogrammetry can be used concurrently with BIM platform (Building Information Modelling) softwares, generating models increasingly intelligent and interactive, which allow manipulation by trials in Augmented Reality (AR) and Virtual Reality (VR). The digital heritage preservation is one of the areas to be benefited with the new possibilities. In this way, this paper presents the results of the research whose purpose is to present some advantages of the digital technologies in the preservation of the historical patrimony from the survey by the capture of the reality with photogrammetry and laser scanning, in integration with BIM, RA and RV. For this, a case study was carried out to illustrate an exploration of those alternatives to the panel model of the modern building located in the Faculty of Architecture and Urbanism at Federal University of Rio de Janeiro - UFRJ. It is understood the technologies importance for the heritage preservation as a paradigm, since it is possible to offer an immersive experience in buildings of interest for preservation, besides the complete registration and integration of documentary information about the historic buildings.
\end{abstract}

Keywords: Digital preservation. BIM. Virtual Heritage.

How to cite this article:

CANUTO, Cristiane Lopes; MOURA, Larissa Ribeiro de; SLAGADO, Mônica Santos. Tecnologias digitais e preservação do patrimônio arquitetônico: explorando alternativas. PARC Pesquisa em Arquitetura e Construção, Campinas, SP, v. 7, n. 4, p. 252-264, dez. 2016. ISSN 1980-6809. Disponível em: <http://periodicos.sbu.unicamp.br/ojs/index.php/parc/article/view/8647456>. Acesso em: 14 maio 2017. doi:http://dx.doi.org/10.20396/parc.v7i4.8647456. 


\section{Introdução}

O processo de projeto das edificações e cidades tem alterado significativamente em função da sua crescente complexidade. $\mathrm{O}$ aumento na quantidade de especialidades (disciplinas) que concorrem para a produção do projeto completo da edificação tem obrigado os coordenadores ao estudo e análise de novas tecnologias visando à compatibilização dos projetos e redução dos erros. Para isso a simulação da construção no meio virtual através de um modelo, como um modelo BIM - Building Information Modeling - ou modelagem da informação da construção, mostra-se eficiente antecipando as decisões projetuais (DANTAS FILHO et al., 2016).

No que se refere aos projetos voltados à preservação do patrimônio histórico e cultural, a complexidade aumenta em função das limitações muitas vezes impostas pela legislação que protege o bem. Paralelamente, observa-se que, frequentemente, a documentação e registro histórico dessas edificações encontram-se distribuídos entre diferentes órgãos e o trabalho de resgate das informações originais torna-se extremamente complexo.

Nos últimos 10 anos, os avanços na área da tecnologia da informação têm revelado possibilidades nunca imaginadas pelos arquitetos e engenheiros civis. Os vetores tecnológicos de mudança, segundo o World Economics Forum (2016) no relatório sobre os empregos do futuro, são:(i) Internet móvel e tecnologia em nuvem, (ii) poder de processamento e Big Data, (iii) novas fontes de energia e tecnologias, (iv) Internet das Coisas, (v) economia compartilhada e crowdsourcing, (vi) robótica e transporte autônomo, (vii) inteligência artificial, (viii) manufatura avançada e impressão 3D e (ix) finalmente materiais avançados e biotecnologia. Sendo que os impactos das tecnologias (i), (ii) e (v) já são sentidos hoje. Os impactos das tecnologias (iii), (iv) e (viii) serão evidenciados até 2017. Os avanços na tecnologia de fabricação digital prometem uma nova onda de produtividade, de 2018 a 2010 passaremos a sentir os efeitos das tecnologias restantes, i.e., (vi), (vii) e (ix). Os avanços na inteligência artificial, aprendizado de máquinas e interfaces de usuário (por exemplo, reconhecimento de voz) estão alterando significativamente os ambientes de trabalho, com a automação de tarefas anteriormente consideradas impossíveis para as máquinas

Entre as alternativas oferecidas ao setor de arquitetura e construção, tem destaque a plataforma BIM, que explicita uma nova forma de desenvolvimento dos projetos, com novas possibilidades desde a fase de concepção até a fase de uso-operação, manutenção e preservação do patrimônio edificado. Sabe-se que a produção de modelos permite a antecipação das decisões projetuais e das soluções construtivas a serem adotadas no canteiro de obras, estimulando a racionalização da construção e a redução de
custos.(EASTMAN, 2011; PEREIRA, 2013) Nesse sentido, a modelagem da informação da construção surge como uma alternativa importante também para a gestão do processo de projeto de restauração e preservação do patrimônio, viabilizando a identificação das incompatibilidades entre as especialidades de projeto e facilitando o processo de construção ou recuperação.

Acrescente-se a essa discussão, as novas possibilidades de interação viabilizadas por esses modelos, à medida que estes podem também ser utilizados nos programas de engenharia de games, permitindo a criação de protótipos que se adéquam as nossas necessidades de interação, manipulação, imersão ou representação. Aplicações em Realidade Aumentada (RA) e Realidade Virtual (RV), podem modificar a maneira de projetar, simular o funcionamento de equipamentos e/ou a presença de pessoas, apresentar o detalhamento do modelo real, viabilizar a análise de futuras intervenções, analisar modificações, ou seja, experimentar o interior de um edifício em qualquer fase de sua produção que se deseje, desde a concepção até a fase de operação e manutenção.

Este artigo apresenta as principais soluções oferecidas, até 2016, pelas tecnologias digitais com ênfase na preservação em arquitetura e demonstra sua utilização por meio de um estudo de caso. O objetivo da pesquisa é apresentar algumas vantagens das tecnologias digitais na preservação do patrimônio histórico a partir do levantamento pela captura da realidade com fotogrametria e escaneamento a laser, em integração com BIM , RA e RV.

Entende-se que a adoção das novas ferramentas pode abrir um novo universo de possibilidades ao processo de preservação do patrimônio, revelando alternativas ainda não exploradas pelo setor.

\section{Possibilidades oferecidas pelas tecnologias digitais}

O ritmo de desenvolvimento e avanço das possibilidades oferecidas pelas tecnologias digitais não tem sido acompanhado pela rapidez no aprendizado e incorporação dessas tecnologias pelas equipes de projetos e obras (KIM et al., 2013; SGUIZZARDI, 2011).

Embora as possibilidades oferecidas pela integração entre modelos BIM e Realidade Aumentada como apoio à execução na construção civil, já tenham sido exploradas por alguns pesquisadores (CUPERSCHMID; GRACHET; FABRICIO, 2015), observa-se certo descompasso entre o que se pode realizar e o que efetivamente tem sido absorvido pelas empresas de projeto, que andam muito presas às técnicas tradicionais de representação gráfica. Pesquisa realizada por Salgado, Cunha e Duarte (2015) revelou o uso tímido da plataforma BIM, mesmo em obras 
com formas arrojadas, tal como o Museu do Amanhã inaugurado na Cidade do Rio de Janeiro.

No que se refere à preservação do patrimônio histórico, observa-se a necessidade de integrar informações que, muitas vezes, encontram-se distribuídas em registros realizados algumas vezes à lápis em papel manteiga, ou apenas em fotos e desenhos artísticos (AMORIM 2010, ABRANTES, 2015; CURSI; SIMEONE; TOLDO, 2015; GROETELAARS, 2015) Nesse particular, técnicas como a fotogrametria e o escaneamento a laser 3D, podem auxiliar na geração de novos dados, que auxiliarão, não apenas na preservação, mas também no resgate das informações projetuais de edificações cujo registro se perdeu no tempo. Destaca-se assim, a importância de discutir e explorar as novas possibilidades tecnológicas aplicadas à preservação do patrimônio histórico.

\section{Panorama sobre o processo BIM}

Os softwares que trabalham na Plataforma BIM permitem a construção de modelos digitais compostos por banco de dados que agregam informações para diversas finalidades, aumentando a produtividade e permitindo a racionalização do processo de projeto e construção (EASTMAN et al., 2011).

Esse novo processo parte não mais de desenhos bidimensionais, mas de modelos tridimensionais $e$ pressupõe que todas as informações relativas à construção, nas diversas fases de seu ciclo de vida, sejam alocadas em um só modelo integrado, paramétrico, intercambiável e passível de simulação, que poderá ser utilizado desde a concepção dos projetos, durante as obras e durante toda vida útil do espaço construído. (AsBEA, 2015a, p.6)

Olin's et al (2012), acrescentam que as vantagens da plataforma BIM não se limitam às fases de concepção do projeto e construção, mas podem ser úteis ao longo de todo o ciclo de vida do edifício, envolvendo as etapas de manutenção, revitalização, a análise do uso do espaço e gestão ambiental, bem como os custos do desempenho operacional. Mas a utilização da nova tecnologia requer, inicialmente, o treinamento das equipes de projeto e o reconhecimento das especificidades deste novo modelo de processo de projeto. Conforme identificado pela AsBEA (2015b), além dos requisitos técnicos do projeto, tais como os sistemas construtivos a serem adotados ou as características de desempenho que o edifício deve atingir, existem os requisitos característicos do processo BIM que incluem a definição do nível de desenvolvimento do modelo (conhecido como LOD) e o nível de detalhamento das informações contidas nos elementos construtivos (definido como LOI) entre outros parâmetros que precisam ser definidos para viabilizar o desenvolvimento do projeto de forma colaborativa.
De acordo com Volk, Stengel e Schultmann (2013), a plataforma BIM esta sendo adotado predominantemente nas fases de projeto e construção de novas edificações, com pouca representação em edificações existentes, principalmente de interesse em preservação.

\section{As publicações explicitamente dedicada ao BIM para edifícios existentes, especialmente sem o modelo BIM preexistente e discutindo desafios de pesquisa relacionados, são raros. (VOLK; STENGEL; SHULTMANN, 2013 p.112, tradução nossa)}

Contudo, esses autores demonstram através de uma análise de publicações de artigos científicos entre 2005 e 2013 um aumento no interesse pela utilização de modelos BIM para representar edificações existentes . Esse interesse é impulsionado com as tendências da digitalização e automação, o crescimento de construções existentes e requisitos de sustentabilidade, somando-se a inserção e popularização de tecnologias como internet móvel, computação em nuvem, web semântica, dentre outros (VOLK; STENGEL; SHULTMANN, 2013).

Cunha, Ribeiro e Salgado (2015) destacaram outra possibilidade que pode ser explorada: a modelagem de edificações existentes, auxiliando o estudo sobre as soluções de projeto adotadas por arquitetos consagrados. Os desafios da modelagem digital de uma edificação existente são ainda maiores, uma vez que trata-se de preparar um modelo "como construído" a partir de registros realizados em AUTOCAD, ou mesmo em papel manteiga e lápis. Os autores aplicaram a tecnologia BIM na modelagem do Centro de Proteção Ambiental da Usina Hidrelétrica de Balbina, importante projeto do arquiteto Severiano Mario Porto. (Figuras 1 e 2) ${ }^{1}$.

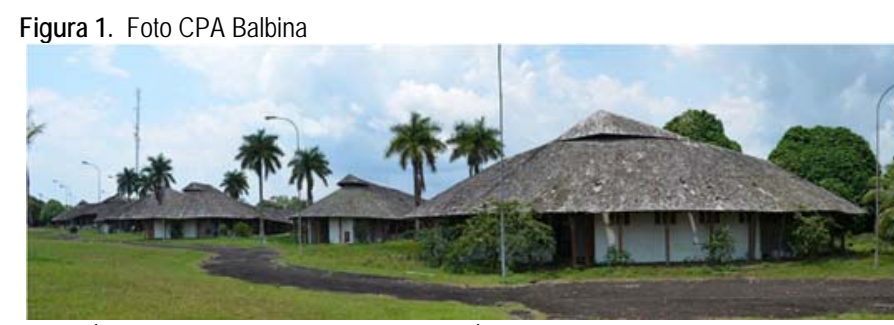

Fonte: (CUNHA ; RIBEIRO; SALGADO, 2015)Figura 2. Modelo digital CPA Balbina

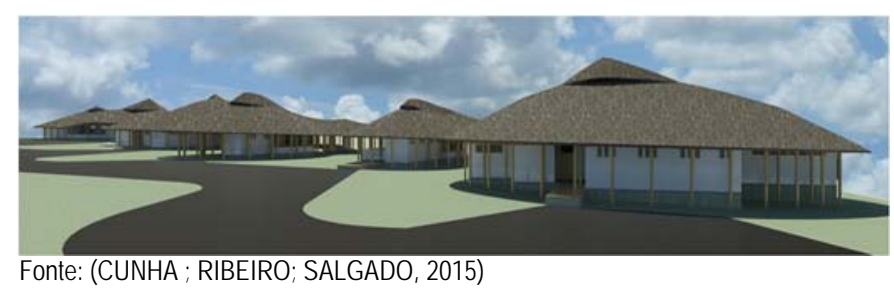

Os autores destacam o potencial da plataforma BIM na preservação da memória arquitetônica e na gestão de facilidades (operação e manutenção) e ressaltaram outras vantagens da modelagem. 
O modelo construído pode ser complementado com as informações referentes aos materiais de construção o que permitiria, por exemplo, a extração de quantitativos de materiais e previsão orçamentária para a recuperação do conjunto (ou parte dele) facilitando sua gestão $e$ preservação.(CUNHA; RIBEIRO; SALGADO, 2015).

\section{Captura da realidade de edifícios históricos e} integração do modelo digital com a tecnologia BIM

Na área de preservação do patrimônio histórico, observase o aumento do uso de ferramentas que permitem a captura da realidade do patrimônio edificado. O levantamento com varredura a laser e fotogrametria 3D, tem se destacado em pesquisas nacionais (AMORIM, 2010; DEZEN-KEMPTER et al. 2015; GROETELAARS, 2015) como opções para levantamento, diagnóstico, documentação, em interação com outras tecnologias ligadas a área de construção, auxiliando na reconstrução precisa do bem e, consequentemente na análise e interpretação das edificações.

Os resultados dessa reconstrução digital como um modelo de informação da construção, podem servir para gerar documentação ou atualizar os desenhos e modelos as-built. Ressalte-se que, além do potencial desse modelos na investigação diagnóstica e conservação de edifícios históricos, as possibilidades de integração com plataformas de imersão virtual e Realidade Aumentada cada vez mais acessíveis - revelam-se como alternativas viáveis tanto para divulgação quanto para a educação patrimonial.

De acordo com Dezen-Kempter et al. (2015), tanto o escaneamento a laser 3D quanto a fotogrametria digital, são tecnologias que permitem através de sensores baseados em ondas de luz para medição, direta e indireta, a captura do estado real de uma edificação histórica de forma precisa e eficiente. Groetelaars e Amorim, (2012) acrescentam que esse tipo de levantamento não seria possível com métodos de pesquisas anteriores, pois essas tecnologias mostram as irregularidades, deformações e desgastes, decorrentes do ciclo de vida da edificação.

Como mostrado no (Quadro 1), essas duas tecnologias de levantamento são bastante distintas, quanto ao custo de equipamento, habilidade, precisão e processo de aquisição tridimensional. O modelo obtido através da técnica da fotogrametria apesar de ter um custo baixo, as informações precisam ser tratadas por um conjunto de softwares, esse processamento somente pode ser realizado após o levantamento fotográfico, diferente do escaneamento a laser 3D que possui uma captura e extração automática para geração do modelo geométrico. No entanto, essas duas técnicas são consideradas como tecnologias que se integram para suprir as lacunas de cada levantamento (DEZEN-KEMPTER et al. 2015).

Quadro 1 - Comparação entre as tecnologias digitais, escaneamento a laser 3D e fotogrametria 3D

\begin{tabular}{|c|c|c|}
\hline TECNOLOGIAS & $\begin{array}{c}\text { ESCANEAMENTO } \\
\text { A LASER 3D }\end{array}$ & $\begin{array}{c}\text { FOTOGRAMENTRIA } \\
\text { 3D }\end{array}$ \\
\hline Precisão & Milímetros & Centímetros \\
\hline $\begin{array}{c}\text { Custo de } \\
\text { equipamento }\end{array}$ & Alto & Médio - Baixo \\
\hline $\begin{array}{c}\text { Habilidade } \\
\text { necessária }\end{array}$ & Alta & Média \\
\hline Portabilidade & Á mão & Pós-procesamento \\
\hline $\begin{array}{c}\text { Geração de dados } \\
\text { 3D }\end{array}$ & Captura automática & Modelagem manual \\
\hline Modelagem 3D & $\begin{array}{r}\text { Extração automática } \\
\text { da forma }\end{array}$ & \multicolumn{2}{|c|}{ (2015) } \\
\hline
\end{tabular}

Fonte: elaborado pelos autores baseado em Klein (2012) e Groetelaars (2015)

Nos últimos anos com a universalização da foto digital e a popularização dos UVAs (Unamed Aerial Vehicle) que são os veículos aéreos não tripulado conhecidos como drone, a fotogrametria passou ser adotada nos levantamentos arquitetônicos com a geração de modelos digitais 3D. Os drones permitem maior alcance de registro, segurança no levantamento, com a possibilidade de fotografar grandes alturas, áreas de acesso restrito e etc.

A utilização de modelos 3D precisos e fotorealístas de edifícios históricos, se destacam na reconstituição e restauração por apresentarem precisão nas características espaciais e geométricas do edifício. Todavia, a possibilidade de agregar informações de diferentes fontes e diferentes áreas, torna-os úteis para diferentes profissionais. Ressalte-se que, quanto maior a integração entre as tecnologias digitais e a interoperabilidade do modelo, maior o seu potencial para documentação, intervenção, manutenção e operação do edifício existente. Brumana et al. (2013) reafirmam a eficiência dos modelos para a restauração, quando se consideram questões referentes aos processos construtivos, aos materiais, e às intervenções sofridas pelo patrimônio ao longo dos anos.
(...) nesse contexto Dezen-Kempter et al (2015) destacam a questão do inventário completo - comprehensive inventory - que seria um modelo tridimensional semântico que poderia funcionar como um catalisador central de informações do ciclo de vida da edificação. Esse inventário armazenaria as informações sobre as formas, detalhes construtivos, o histórico e contexto do edifício, e poderia ser construído utilizando a Plataforma BIM. (CANUTO; SALGADO, 2016).

Diante das tecnologias mencionadas tem destaque $\mathrm{o}$ recente termo sobre modelos BIM na área do patrimônio histórico: o HBIM (Historic Building Information Modeling) que é segundo Murphy, McGovern e Pavia (2007) o modelo BIM desenvolvido a partir de dados 
históricos, escaneamento a laser e fotogrametria. Os trabalhos sobre HBIM ilustram como uma biblioteca de objetos paramétricos interativos podem ser criadas. Diferentes tipos de manuais arquitetônicos são usados para obter as dimensões, proporções e informações sobre construção geométrica (DORE; MURPHY, 2013).

Paralelamente à questão do modelo da informação da construção, pode-se integrar ao modelo experiências de RA e RV, durante o processo e após o modelo ser concretizado. As experiências RA e RV podem auxiliar para no processo de modelagem, intervenção na divulgação do patrimônio em áreas como o turismo e a educação patrimonial.

\section{Virtualidade e preservação do Patrimônio}

As discussões apresentadas até aqui, revelam as possibilidades de integração das tecnologias digitais ao desenvolvimento dos projetos arquitetônicos, permitindo a criação de modelos cada vez mais fidedignos, que permitem ampliar as possibilidades de atuação profissional na fase de uso-operação e manutenção. Além disso, as novas tecnologias facilitam o acesso às informações sobre os bens culturais através das novas tecnologias para registro, reconhecimento, valorização e promoção de patrimônios históricos, criando novas formas de visualização, manipulação e interação com os modelos e registros documentais.

Há uma diferenciação entre "Digital” e "virtual”, encontramos objetos digitais no mundo real e no virtual, mas, o mundo virtual se limita a objetos digitais. Digitalizar dados permite seu acesso via rede, em tempo real, contribui com a preservação do patrimônio, seja com a criação de ambientes virtuais, bibliotecas digitais, documentos eletrônicos, hipertextos, ou pela possibilidade de realizar visitas virtuais interativas, entre outros (RIMKUS, 2013).

A virtualização do patrimônio está cada vez mais abrangente, com tecnologias que permitem experimentações em RV e RA- muitas ferramentas vêm se desenvolvendo nas últimas décadas e permitem a visualização e a simulação de ambientes imersivos (RV) e semi-imersivos (RA) de interação, seja de bens existentes, ou não, podendo ser aplicadas no estudo e divulgação do patrimônio cultural. A Realidade Aumentada se fundamenta na transposição de objetos virtuais no espaço físico do usuário, permitindo uma interação em tempo real, tornando mais sutil a relação de conteúdos digitais com o meio. A potencialidade da RA na preservação e restauro do patrimônio é notável. Segundo Rimkus (2013), a realidade aumentada possibilita visualizar o edifício, e compreender os usos e funções ao longo do tempo e também compreender a sua interação com o meio urbano em que está inserido.
Realidade virtual pode ser definida como um ambiente composto por simulações computacionais interativas que identificam o posicionamento do participante e suas ações, e permite uma ação desse indivíduo no meio em que está inserido, dando a sensação de estar mentalmente imerso na simulação. Entre os dispositivos que permitem essa experiência cita-se o head-mounted display (HMD), uma espécie de capacete onde imagens gráficas são exibidas em uma tela ou um par de telas (uma para cada olho). Um sensor de rastreamento conectado à cabeça do participante informa ao sistema de computador o posicionamento do participante bem como a direção para onde está olhando. (SHERMAN; CRAIG, 2003, tradução nossa).

Entre as possibilidades oferecidas pela da interação das tecnologias digitais com o patrimônio, é possível observar vantagens no planejamento de ações de proteção do patrimônio e na criação de registros documentais na preservação da memória.

É comum encontrar páginas na internet que permitem visitas virtuais de bens de interesse, viabilizando, por exemplo, o acesso à galerias de exposição com fotos esféricas e vídeos em $360^{\circ}$. A divulgação dos monumentos em Patrimônio Virtual através da rede, permite atingir a um público infinito, viabilizando a visita à distância, ou mesmo a experiência de conhecer virtualmente bens que não mais existem no mundo físico (pela destruição provocada por guerras, catástrofes ou intempéries, entre outros) .

Tais simulações necessitam de modelagens tridimensionais, que podem ser facilitadas pelas novas tecnologias, gerando modelos com as mais variadas possibilidades de interação, baseados em: registros gráficos, documentos e levantamentos de todo o conjunto, de seu entorno, plantas arquitetônicas iniciais e de modificações ao longo do tempo, fotografias e registro de modificações ao longo dos anos, entre outros. Esses registros permitem a melhor compreensão do monumento explorado, podendo ser utilizados na educação patrimonial e no turismo virtual (NOGUEIRA, 200-?).

Experiências imersivas estão em constante desenvolvimento e sua popularização tem sido viabilizada graças às plataformas com custo mais acessível, como os óculos de Realidade Virtual (presentes hoje no mercado), que tem boa capacidade de processamento. Estes óculos deixam o usuário imerso em modelos quase fotorrealísticos (dependendo do dispositivo utilizado), seja através de equipamentos conectados a computadores ou acoplados a celulares. Essa tecnologia tende a expandir a noção de realidade e está cada vez mais presente no mercado, como o Google CardBoard (futuramente Google Daydream - Figuras 3), o Samsung GearVR e o Oculus Rift (Figura 4), entre outros. 


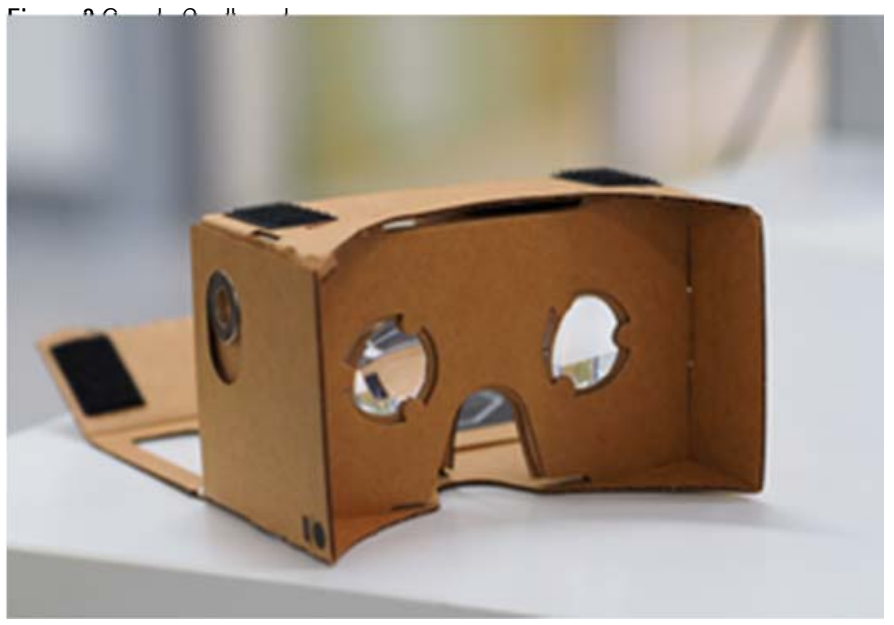

Fonte:https://commons.wikimedia.org/wiki/File\%3AAssembled_Google_Cardboard_VR _mount.jpg).

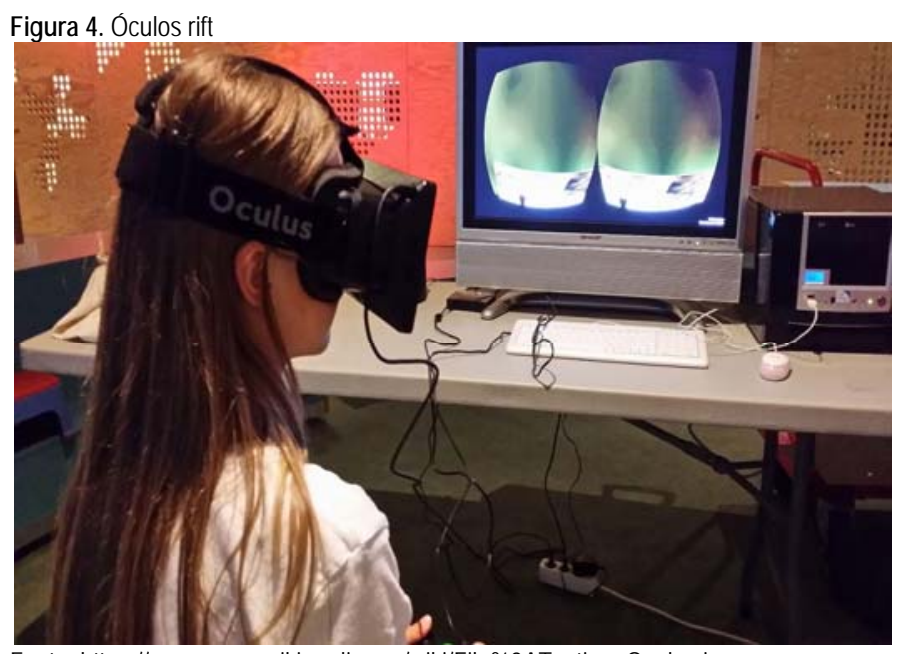

Fonte: https:// commons.wikimedia.org/wiki/File\%3ATesting_Oculus.jpg

O uso dessa tecnologia na Arquitetura permite, por exemplo, a apresentação dos projetos aos clientes através de um passeio virtual pelo modelo, viabilizando a visualização e interação com o projeto, gerando a sensação de habitar um espaço sem que o usuário esteja fisicamente presente, ou mesmo para visualizar decisões de projeto no momento de sua criação ou intervenção.Programas específicos permitem a visualização em tempo real dos modelos editados nos programas. A implementação da tecnologia é facilitada pela compatibilização entre programas de engenharia de games (os mesmos utilizados na criação de jogos de videogame) com softwares habitualmente utilizados por arquitetos como o SketchUp, Blender, 3DSmax, Archicad e Revit.
O CardBoard é um dos modelos de menor custo do mercado, pode ser construído a partir de um guia de especificações e oferece a possibilidade de ser utilizado junto a um celular do tipo SmartPhone (celulares inteligentes equivalentes a computadores), tornando-se um dispositivo portátil capaz de conquistar um setor anteriormente inacessível (com produtos como o Oculus Rift - que tem um preço mais elevado por proporcionar maior qualidade de imersão e envolvimento com ambiente virtual). O molde do CardBoard está disponível online e pode ser montado por qualquer pessoa, permitindo uma experiência em realidade virtual simples, interativa e barata, que utiliza softwares de código aberto e fornece resultados consideráveis. A tendência é pela contínua popularização dos mecanismos e softwares, que permitirão, em última instância, a experiência imersiva em RA e RV a qualquer pessoa que possua um smartphone e um óculos de RV.

\section{Preservando o patrimônio moderno: estudo de caso}

O edifício que hoje abriga a Faculdade de Arquitetura e Urbanismo da Universidade Federal do Rio de Janeiro, foi projetado pelo arquiteto Jorge Machado Moreira e construído na década de 50, traduzindo as características modernistas em suas qualidades técnicas, arquitetônicas e paisagísticas. A obra foi concebida seguindo princípios da arquitetura moderna do arquiteto Le Corbusier, apresentando harmonia na forma, um bloco vertical que sobrepões a um bloco horizontal e aspectos de racionalidade construtiva, evidenciado desde à estrutura ao seu acabamento.(Figura 5).

O paisagismo e o painel da fachada do bloco horizontal foram projetados pelo paisagista Burle Marx e refletem as marcas do modernismo brasileiro. Apesar de toda racionalidade do edifício, Burle Marx acrescenta um toque artístico com seus jardins orgânicos com plantas nativas do Brasil e com o painel em concreto armado na fachada do edifício. O painel possui 53,88 m de comprimento em sua face reta por $10,25 \mathrm{~m}$ de altura e apresenta uma pequena curvatura na face exterior, a qual é repleta de diferentes ornamentos ortogonais, com diferentes profundidades, sendo como uma escultura do edifício. As qualidades modernistas do prédio da FAU resistem ao longo tempo, entretanto o edifício está em estado crítico de degradação. 


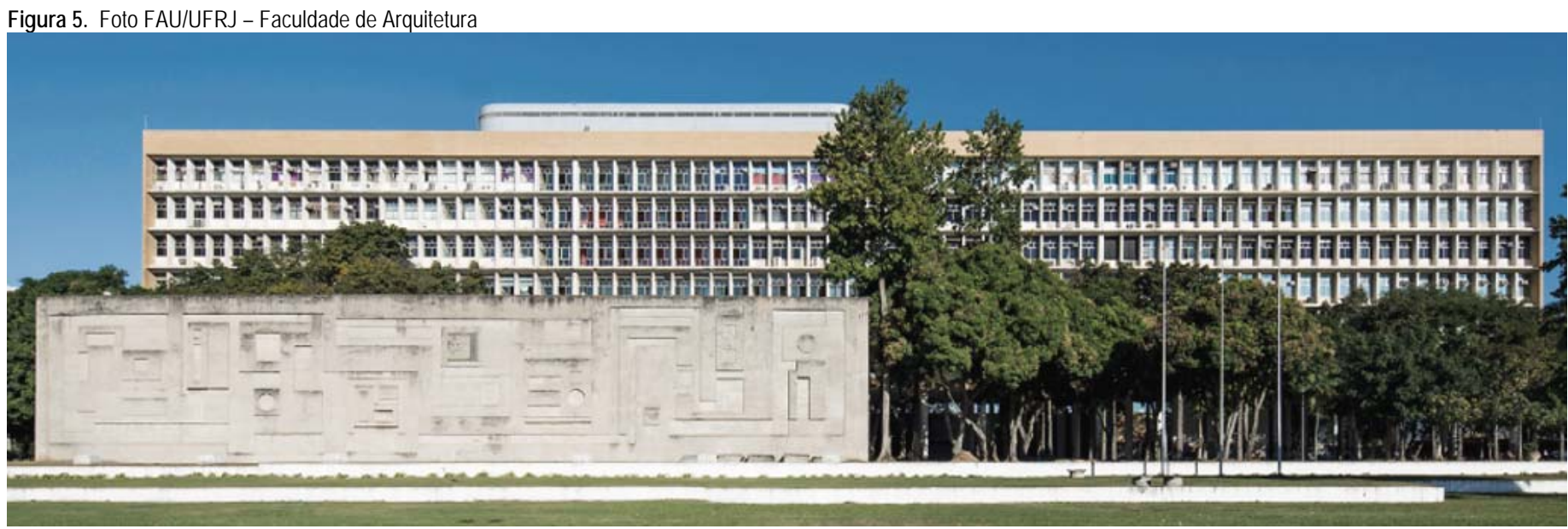

Fonte: os autores

Avançando para a prática, diante da abordagem conceitual apresentada sobre a importância das tecnologias digitais para a preservação do patrimônio histórico, ilustra-se um estudo de caso de um importante elemento do prédio da FAU, o modelo BIM do painel e a possibilidade do uso deste modelo para RV e RA. O processo ocorreu conforme ilustra a (Figura 6).
O estudo de caso foi motivado pelo levantamento do painel da FAU com a técnica de escaneamento a laser 3D ,o qual foi realizado como atividade da disciplina de Mestrado no segundo semestre de 2015. O resultado foi o modelo geométrico em nuvem de pontos do painel da FAU. Os especialistas em tecnologia em escaneamento a laser, Carlos Coutinho e Kezio Arruda foram responsáveis pelo levantamento demonstrando e explicando a tecnologia.

Figura 6. Sequência das atividades relacionadas ao estudo de caso

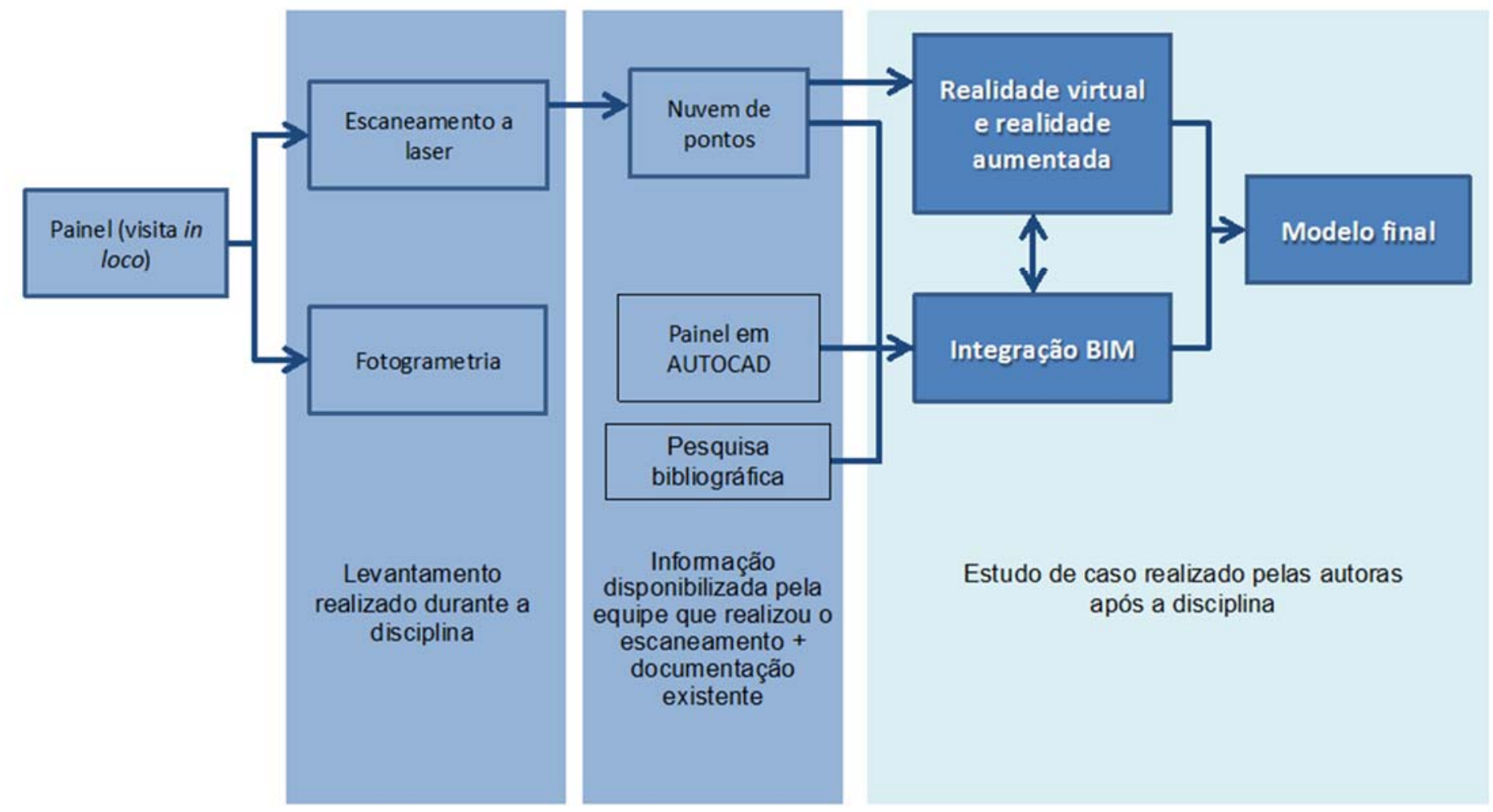

Fonte: os autores 
Figura 7. - Ortofoto a partir da nuvem de pontos do painel do prédio FAU - UFRJ

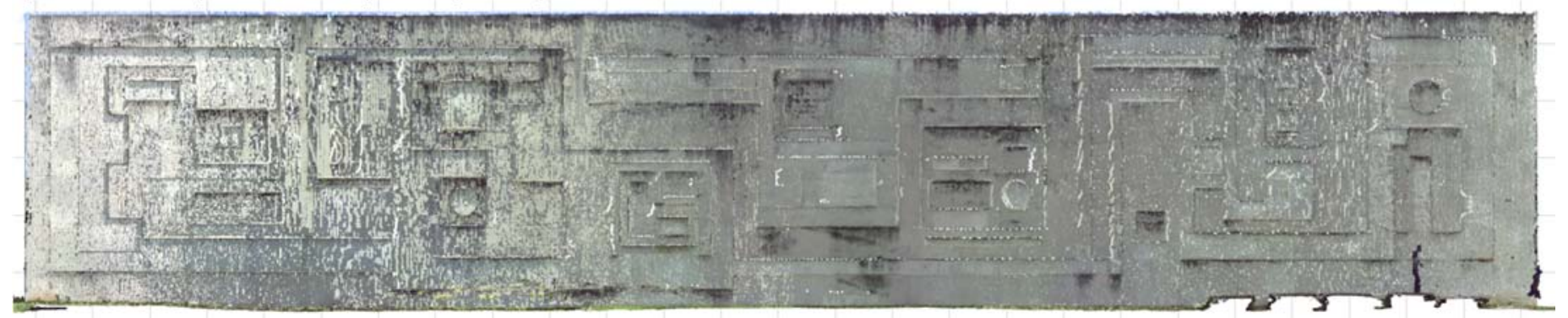

Fonte: os autores

O equipamento utilizado foi o Laser Escâner Terrestre (LET) Faro Focus 330x. Segundo a fabricante FARO , esse equipamento possui uma velocidade de escaneamento de um 976 mil por segundo em uma distância de até 330 metros. Ele pode operar em temperaturas que variam de 5 graus Celsius até 40 graus Celsius. Um equipamento ideal para grandes e complexas construções, com um tempo de levantamento curto em relação a levantamentos manuais e com bom desempenho diante de variações de temperatura. Dependendo da obra a ser levantada, pode existir a necessidade de associar essa tecnologias a outras, como os drones - para acesso a regiões de difícil alcance - e fotogrametria - para cobrir falhas na nuvem adquiridas pelo escaneamento a laser. As barreiras físicas também são outro aspecto a considerar no planejamento desse levantamento.

O levantamento do painel do prédio da FAU foi feito com 6 cenas (posições do scanner). De acordo com os especialistas as cenas foram planejadas e posicionadas de modo que uma sobrepusesse partes da outra, para permitir registro (união das cenas) sem que ocorressem lacunas no levantamento. Após a aquisição dos dados estes foram transferidos para um computador e tratados no software Faro Scene, onde além dos pontos adquiridos foi acrescentada cor real ao levantamento, utilizando as imagens que o aparelho laser escâner captura através de uma câmera integrada. Ao final do processo, a nuvem de pontos tridimensional foi gerada e com condições de exportação em vários formatos interpretáveis em softwares CAD - Computer Aided Design e CAE Computer Aided Engineering. Toda essa etapa foi realizada em menos de duas horas.

O resultado, de maneira geral, atendeu aos objetivos da disciplina, todavia, observa-se que, para a plenitude do modelo da nuvem de pontos, seria necessário levantar uma parte em outro horário localizada a esquerda da fachada do painel, pois o escaneamento apresentou algumas falhas em decorrência da posição do sol. Outra alternativa seria suprir essa lacuna utilizando o recurso da fotogrametria (Figura 7). A adoção da tecnologia a laser foi essencial para esse caso específico, pois o painel encontra-se em estado de conservação comprometido, e possui certa complexidade na forma, pelos seus diferentes ornamentos e pequena curvatura.

A segunda etapa, realizada após a disciplina e consistiu na demonstração da possibilidade de "adicionar inteligência" ao modelo de nuvem de pontos - termo usado por teóricos do HBIM - ou seja, adicionar informações que vão além da superfície levantada utilizando a plataforma BIM (MURPH; McGOVERN; PAVIA, 2013). Para isso dados construtivos e técnicas, foram adquiridas através de levantamento CAD 2D e documentação existentes.

A nuvem de pontos pode ser extraída em vários formatos a partir do software Faro Scene. No estudo de caso o formato utilizado foi o .e57 - LIDAR Point Cloud Data esse formato é usado para armazenar dados capturados por sistemas de imagem 3D e é compatível com o Archicad 19 da Graphisoft, software utilizado para a criação de modelos BIM (Figura 8). A modelagem poderia ser feita utilizado outros softwares BIM , como Revit da Autodesk, contudo o arquivo para importação deve estar em formatos .rcs -Random Control Scene ou .rcp -Rich Chart Builder Project.

Figura 8. Imagem do painel da FAU UFRJ importado a partir da tecnologia do escaneamento a laser 3D

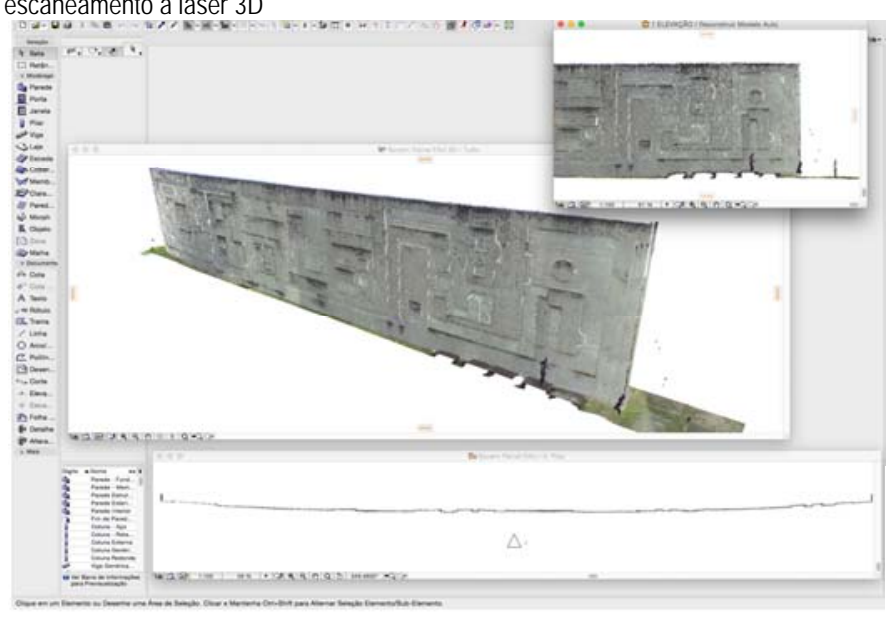

Fonte: os autores

Após a importação da nuvem de pontos alguns cortes longitudinais foram realizados para obter as dimensões dos elementos que compõe o painel, os quais não constam no levantamento existente em CAD 2D (Figura 8). Com a 
integração entre captura da realidade 3D do painel, software BIM e documentação existente, foi possível aumentar o potencial de informações do modelo que apesar de ser apenas um elemento do edifício, representa o começo de uma biblioteca de objetos paramétricos que vão fazer parte do modelo histórico BIM do prédio da FAU.

As maiores dificuldades encontradas na literatura as quais referem-se a modelagem BIM a partir de modelos fotorealísticos são: modelagem de formas irregulares, manipulação da nuvem pelo tamanho dos arquivos que são gerados para importação, perda de dados na importação, determinação do nível de detalhe do modelo e falta de componentes adequados para modelar edificações históricas em softwares BIM. No estudo de caso, essas dificuldades foram estudadas e em sua maioria vencidas, primeiro a nuvem a ser importada é de um elemento e não da edificação como um todo, viabilizando sua manipulação, depois a importação da nuvem em formato e.57 para o Archicad 19 não houve perda de informações nem textura e por fim os ornamentos foram modelados utilizando a ferramenta morph do próprio programa. Entretanto, a modelagem foi baseada em trabalho manual, tanto a detecção de medidas na nuvem de pontos quanto para modelagem paramétrica, mostrando a necessidade de automação de reconhecimento de nuvem de pontos e interação de dados no processo.

Figura 9. Modelo do painel FAU - UFRJ, em nuvem de pontos e BIM

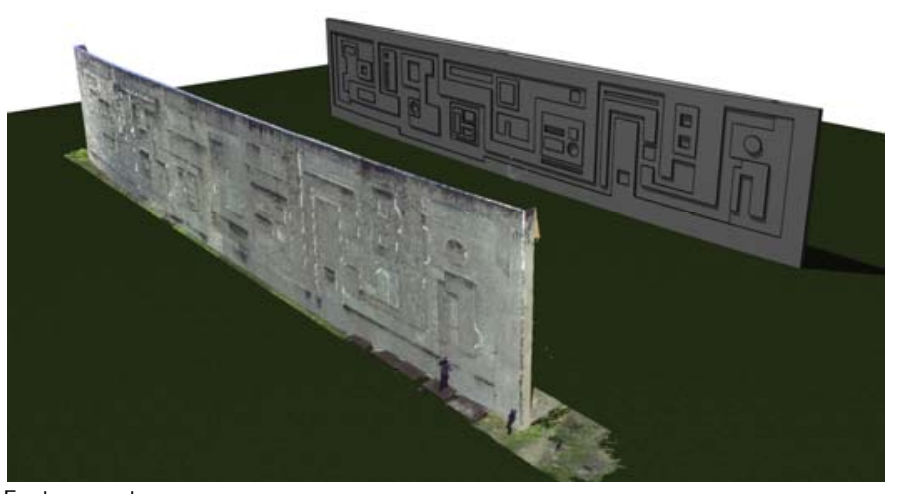

Fonte: os autores

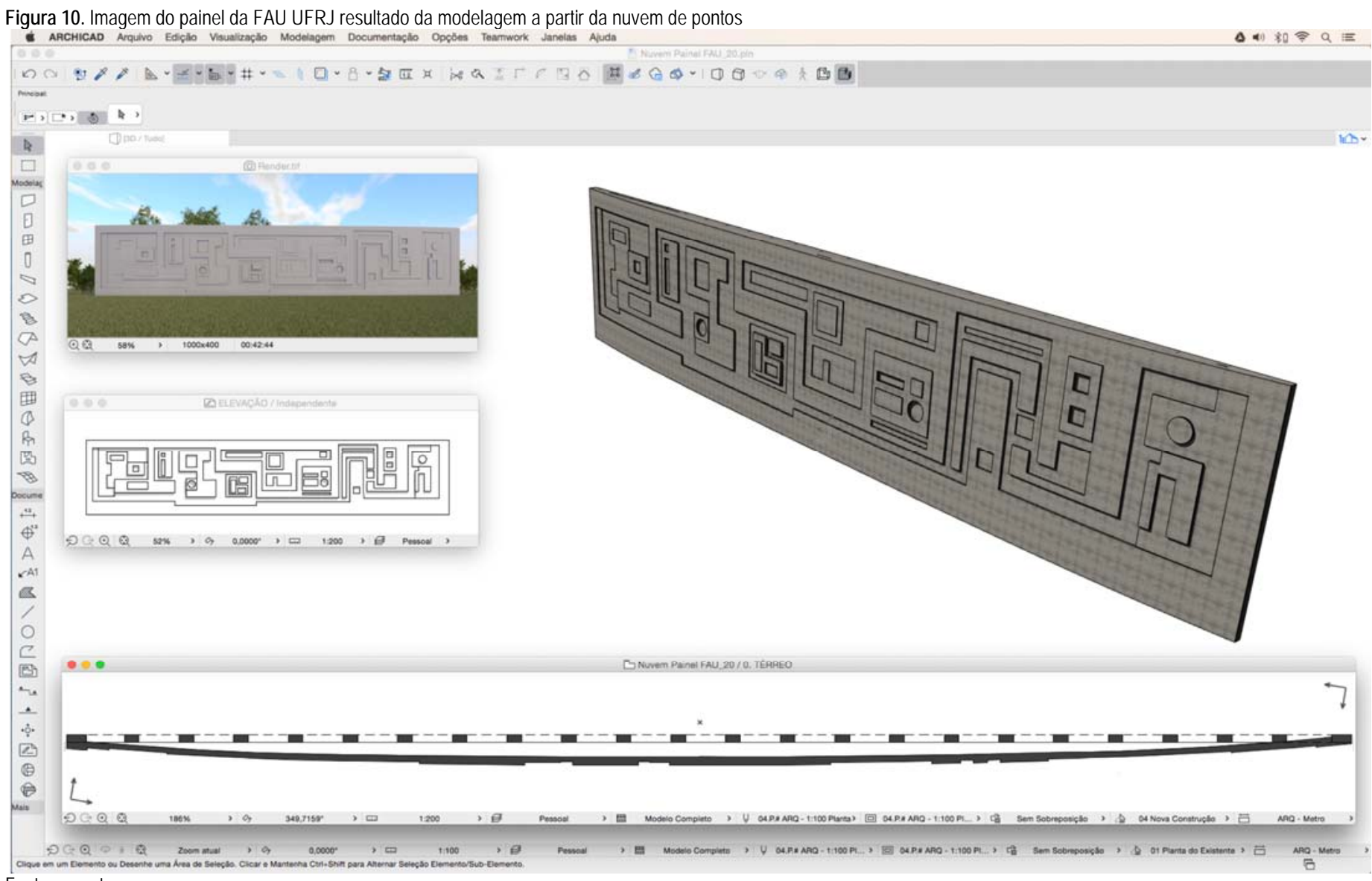

Fonte: os autores 
O resultado dessa etapa mostra um modelo BIM que graficamente possui as geometrias dos seus elementos, com dimensões, formas, quantidade e localização que refletem suas condições próximas a realidade, com desvio máximo de $20 \mathrm{~mm}$ da nuvem para a modelagem BIM (Figura 10). O importante nessa etapa do estudo de caso foi avaliar como o modelo BIM possibilita a transição do conceito de superfície para representação de objeto.

A terceira etapa do experimento consistiu na aplicação da Realidade Aumentada, com o objetivo de viabilizar a visualização e manipulação da modelagem in loco, considerando as coordenadas de geolocalização (com precisão milimétrica) ou através de rastreamento de imagem (código) via celular. A visualização em RA foi validada com o modelo em escala. Com a utilização de um aplicativo (Augment), o celular identifica o código que permite uma análise e controle de visualização do modelo via toque na tela do aparelho (Figura 11). O objeto virtual, pode ser inserido no meio físico do usuário da tecnologia, seja alocando a modelagem em sua posição real no globo, ou rastreando a imagem a partir da planta impressa em papel e sobrepondo o modelo exatamente em sua posição.

Com o objetivo de avaliar a possibilidade de utilização do modelo em experiências imersivas de Realidade Virtual, o objeto virtual do painel da FAU, foi importado para um software de engenharia de games, o Unity $3 \mathrm{D}$, que cria uma ambientação para o usuário dos óculos de RV (Figura 12).

A simulação permite que o usuário passe a interagir como uma personagem (em primeira pessoa) imerso em um mundo virtual onde estará locada a modelagem de simulação do painel da FAU - quanto melhor trabalhado e programado o modelo, maior a capacidade de imersão, interação e envolvimento, da pessoa com o meio. Além dessa capacidade de vivência de um espaço imaginário, interações podem ser programadas, dados podem ser incorporados (entre outras possibilidades), o modelo é configurado para ser visualizado em óculos de RV.

A tecnologia se torna uma ferramenta de manipulação de formas cada vez mais complexas de serem geradas e mais simples de serem visualizadas e compreendidas, com qualidade cada vez mais fotorealística, podendo ser agregada ao campo do patrimônio, como um facilitador na análise de dados e na criação de experiências imersivas para os mais diversos fins, como passeios virtuais, estudos de revitalização, restauração, projetos de intervenção, vivência de ambientes que já não existem mais fisicamente, entre outras possibilidades.

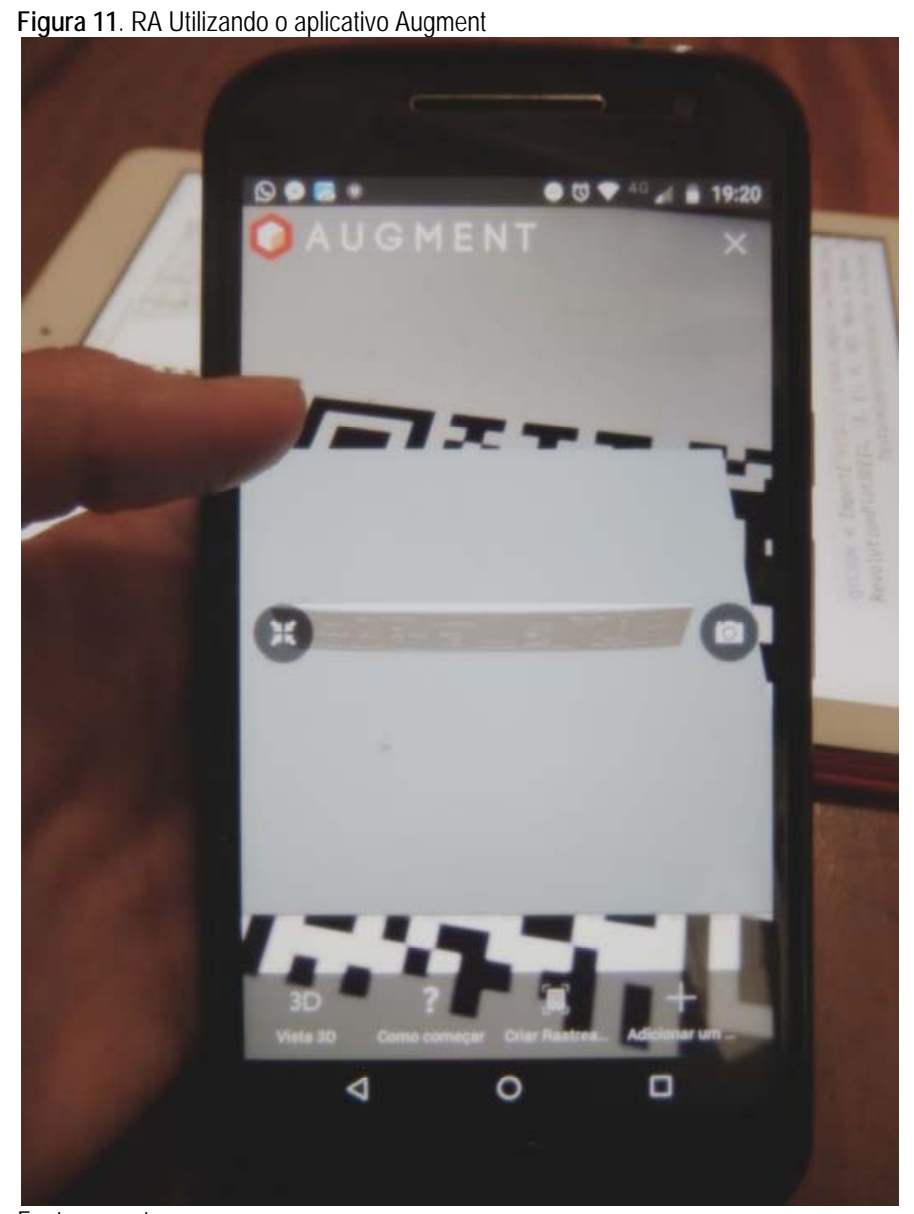

Fonte: os autores

Figura 12. Modelagem inserida no motor de jogos e programada para visualização em óculos de RV (Cardboard).

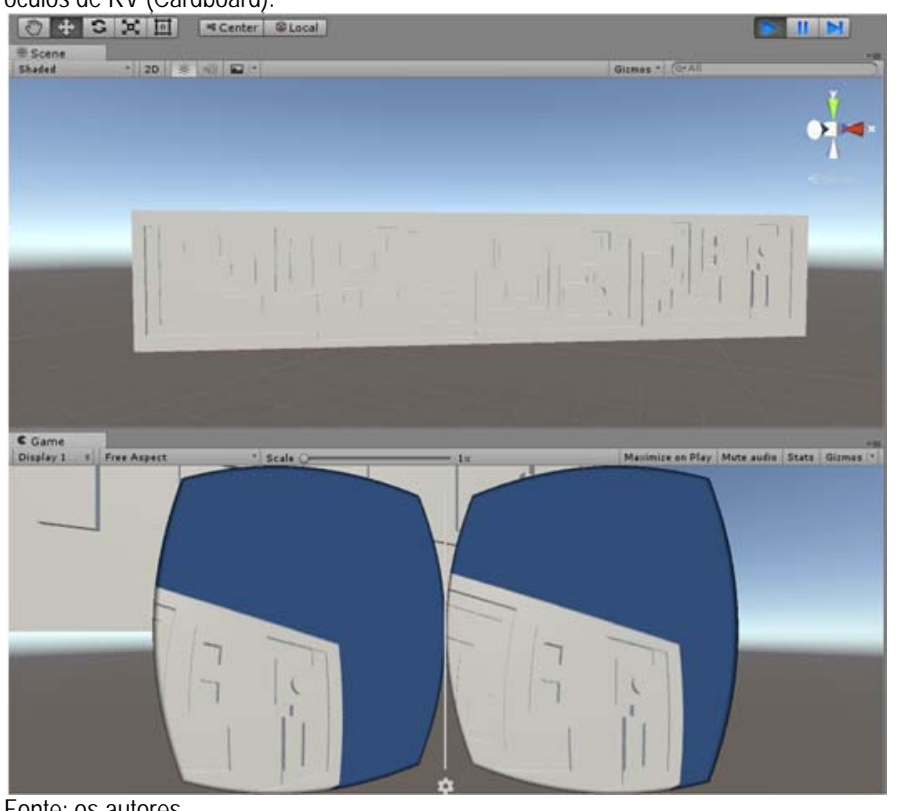

Fonte: os autores

\section{Conclusão}

Esse artigo apresentou as principais possibilidades oferecidas pelas tecnologias digitais à área da preservação do patrimônio arquitetônico, considerando o avanço do conhecimento até a presente data - 2016. 
No que se refere à plataforma BIM, observa-se seu potencial não como repositório de informações, mas como instrumento para a gestão da manutenção e preservação das edificações, auxiliando os gestores na fase de usooperação e manutenção a partir do compartilhamento de dados com outros softwares.

A fotogrametria e o escaneamento em 3D, por sua vez, revolucionaram a realização de levantamentos e diagnósticos do bem, a partir da agilização no processo de captura e tratamento das imagens, abrindo, portanto, outras possibilidades de estudos sobre o patrimônio construído. Finalmente, a realidade aumentada aproxima a informação do usuário, facilitando o acesso e interpretação de informações, enquanto a realidade virtual oferece a possibilidade de transportar o usuário para um ambiente de simulação. Essa tecnologia permite, por exemplo, oferecer ao usuário a experiência de vivenciar o espaço de edificações que se encontrem parcial ou totalmente destruídas.

As alternativas tecnológicas aqui apresentadas devem ser avaliadas pelos arquitetos de forma a serem utilizadas considerando não apenas os custos de implantação, mas especialmente os resultados positivos que tais métodos podem trazer à preservação da memória arquitetônica. Ressalte-se que este artigo não teve a intenção de esgotar o tema - que permanece em constante evolução - mas apresentar as principais alternativas, dando visibilidade às possibilidades à disposição dos profissionais interessados nas tecnologias digitais e suas aplicações em arquitetura, engenharia e preservação.

\section{Agradecimentos}

Os autores agradecem ao CNPq pelo apoio à pesquisa (Bolsa de Produtividade em Pesquisa)

\section{Notas}

(1) As visitas ao modelo completo podem ser realizadas a partir do site http://marcoabcunha.wixsite.com/balbinadigital.

\section{Referências}

ABRANTES, Andreza Rigo. Tecnologias digitais como instrumentos de preservação do patrimônio urbano edificado. 2014. 171 f. Dissertação (Mestrado em Preservação do Patrimônio Cultural) - Instituto do Patrimônio Histórico e Artístico Nacional, Rio de Janeiro, 2014.

AMORIM, Arivaldo Leão. Patrimônio Virtual e História Digital: Essência e representação. A Documentação Digital do Patrimônio Construído: Possibilidades e desafios. In: ENCONTRO NACIONAL DA ASSOCIAÇÃO NACIONAL DE PESQUISA E PÓSGRADUAÇÃO EM ARQUITETURA E URBANISMO, 1., 2010, Rio de Janeiro. Anais... Rio de Janeiro:ANPARQ, 2010. p. 125.

AsBEA - ASSOCIAÇÃO BRASILEIRA DOS ESCRITÓRIOS DE ARQUITETURA. Guia de Boas Práticas em BIM. Estruturação do escritório de projeto para a implantação do BIM vol.1, 2016a. Disponível em

<http://www.asbea.org.br/asbea/assuntos/manuais.asp. Acesso em: 15 abril 2016

AsBEA - ASSOCIAÇÃO BRASILEIRA DOS ESCRITÓRIOS DE ARQUITETURA. Guia de Boas Práticas em BIM. Fluxo de projeto em BIM: planejamento e execução vol.2, 2016b. Disponível em < http://www.asbea.org.br/asbea/assuntos/manuais.asp> Acesso em: 15 abril 2016

BRUMANA, R.; ORENI, D.; CUCA, B.; BINDA, L.; CONDOLEO, P.; TRIGGIARI, M., Strategy for integrated surveying techniques finalized to interpretive models in a byzantine church, Mesopotam, Albania. International Journal of Architectural Heritage. v. 8, n. 6, out. 201. doi: http://dx.doi.org/10.1080/15583058.2012.756077

CANUTO, C.L.; SALGADO, M.S. Modelagem da informação da construção na preservação da arquitetura moderna. In: ENCONTRO NACIONAL DE TECNOLOGIA DO AMBIENTE CONSTRUÍDO, 16., 2016, São Paulo. Anais... Porto Alegre: ANTAC, 2016. p. 4864-4875.Disponível em: http://www.infohab.org.br/entac/2016/ENTAC2016_paper_77.pdf. Acesso em: out. 2016.

CUNHA, M. A. B.; RIBEIRO, E.; SALGADO, M. S.. Reconstrução digital da Vila Balbina: preservando o projeto de Severiano Mario Porto. In: ENCONTRO DE TECNOLOGIA DE INFORMAÇÃO E COMUNICAÇÃO NA CONSTRUÇÃO, 7. 2015. Recife. Anais ... Porto Alegre: ANTAC, Blucher, 2015. p. 537-548. doi: http://dx.doi.org/10.5151/engpro-tic2015-049 
CUPERSCHMID, A. R. M.; GRACHET, M. G.; FABRICIO, M. M. Realidade Aumentada como auxílio à montagem de parede em wood-frame. PARC Pesquisa em Arquitetura e Construção, Campinas, SP, v. 6, n. 4, p. 266-276, dez. 2015. ISSN 1980-6809. Disponível em: <http://periodicos.sbu.unicamp.br/ojs/index.php/parc/article/view/8640947>. Acesso em: 27 de dez. 2016. doi: http://dx.doi.org/10.20396/parc.v6i4.8640947

CURSI, S.; SIMEONE, D.; TOLDO, I.. A Semantic Web Approach for Built Heritage Representation. In: CELANI G., SPERLING D., FRANCO J. (Eds.). Computer-Aided Architectural Design Futures. The Next City - New Technologies and the Future of the Built Environment. Communications in Computer and Information Science book series (CCIS, volume 527). Berlin,Heidelberg: Springer, 2015. p. 383-401. doi:http://dx.doi.org/10.1007/978-3-662-47386-3_21

DANTAS FILHO, João Bosco Pinheiro et al. Classificação e distribuição de Requisições De Informação baseadas em BIM: dois estudos de caso de torres residenciais. PARC Pesquisa em Arquitetura e Construção, Campinas, SP, v. 7, n. 2, p. 75-88, jun. 2016. ISSN 1980-6809. Disponível em: <http://periodicos.sbu.unicamp.br/ojs/index.php/parc/article/view/8646358>. Acesso em: 28 março 2017.

DEZEN-KEMPTER, Eloisa et al. ESCANEAMENTO 3D A LASER, FOTOGRAMETRIA E MODELAGEM DA INFORMAÇÃO DA CONSTRUÇÃO PARA GESTÃO E OPERAÇÃO DE EDIFICAÇÕES HISTÓRICAS. Gestão \& Tecnologia de Projetos, Brasil, v. 10, n. 2, p. 113-124, nov. 2015. ISSN 1981-1543.. doi:http://dx.doi.org/10.11606/gtp.v10i2.102710.

DORE, C., MURPHY, M.. Semi-automatic modelling of building facades with shape grammars using historic building information modelling. The International Archives of Photogrammetry, Remote Sensing and Spatial Information Science, v. XL-5/W1 , p.57-64, 2013. doi: http://dx.doi.org/10.5194/isprsarchives-XL-5-W1-57-2013.

EASTMAN, Chuck et al. BIM Handbook: A guide to Building Information Modeling for Owners, Managers, Designers, Engineers, and Contractors. New Jersey: John Wiley \& Sons, 2011.

GROETELAARS, N. J. Criação de modelos BIM a partir de "nuvens de pontos": estudo de métodos e técnicas para documentação arquitetônica. 2015. 372 f. Tese (Doutorado em Arquitetura e Urbanismo) - Faculdade de Arquitetura, Universidade Federal da Bahia, Salvador, 2015.

GROETELAARS, N. J.; AMORIM, A. L. Um panorama sobre o uso de nuvens de pontos para criação de modelos BIM. In: SEMINÁRIO NACIONAL DE DOCUMENTAÇÃO DO PATRIMÔNIO ARQUITETÔNICO COM O USO DE FERRAMENTAS DIGITAIS, 2., 2012, Belém. Anais... Belém: UFPA, 2012.

MURPHY, M.; MCGOVERN, E.; PAVIA, S. Parametric Vector Modelling of Laser and Image Surveys of 17th Century Classical Architecture in Dublin. In: INTERNATIONAL SYMPOSIUM ON VIRTUAL REALITY, ARCHAEOLOGY AND CULTURAL HERITAGE - VAST2007, 8., 2007, Brighton. Proceedings... Brighton: Eurographics, 2007. Disponível em: http://www.riegl.co.at/uploads/tx_pxpriegldownloads/project_2006_pdf. Acesso em: out. 2016

MURPHY, M., McGOVERN, E., PAVIA, S., 2013. Historic Building Informatio Modelling - Adding intelligence to laser and image based surveys of European classical architecture. Journal of Photogrammetry and Remote Sensing, v. 76, p. 89-102, feb. 2013. ISSN 0924-2716 doi:https://doi.org/10.1016/j.isprsjprs.2012.11.006

PEREIRA, Ana Paula Carvalho. A adoção do paradigma BIM em escritórios de Arquitetura em Salvador - BA. $2013.201 \mathrm{f}$. Dissertação (Mestrado em Arquitetura e Urbanismo) - Faculdade de Arquitetura, Universidade Federal da Bahia, Salvador, 2013

KIM, M.J. et al. Virtual Reality for the built environment: a critical review of recent advances. Journal of Information Technology in Construction.v. 16, p. 279-305, ago. 2013. Disponível em: http://www.itcon.org/2013/14. Acesso em: 2 abril 2017

KLEIN, L.; LI, N.; BECERIK-GERBER, B. Imaged-based verification of as-built documentation of operational buildings. Automation in Construction, v. 21, p. 161-171, jan. 2012. doi: https://doi.org/10.1016/j.autcon.2011.05.023.

NOGUEIRA, A. A. M. Estudos: Realidade Virtual (RV) e Virtual Heritage (VH). [S.1.], 200-?. Disponível em: http://www.diretorio.ufrj.br/aurelionogueira/estudos/. Acesso em: 06 março 2016.

OLIN, J. et al.. Virtuality: What does it means for FM?. In: JOINT CIB W070, W092 \& TG72 INTERNATIONAL CONFERENCE ON FACILITIES MANAGEMENT, PROCUREMENT SYSTEMS AND PUBLIC PRIVATE PARTNERSHIP, 2012, Cape Town. Proceedings ... Cape Town: CIB, 2012. p. 20 - 26. Disponível em: http://www.irbnet.de/daten/iconda/CIB_DC24053.pdf . Acesso em: 20 de abril 2016 
RIMKUS, C.. AVALIAÇÃO DA APLICABILIDADE DA TECNOLOGIA DA REALIDADE AUMENTADA NA ÁREA DO PATRIMÔNIO ARQUITETÔNICO. Revista GEINTEC - Gestão, Inovação e Tecnologias, v. 3, n. 2, p. 70-80, jun. 2013. ISSN: 2237-0722. doi:https://doi.org/10.7198/S2237-0722201300020006.

SALGADO, M.S. CUNHA, M. A. B. DUARTE, T. M. P.. Desenvolvimento de projetos sustentáveis usando a plataforma BIM: estudo de caso na Cidade do Rio de Janeiro In: EURO ELECS LATIN AMERICAN AND EUROPEAN CONFERENCE ON SUSTAINABLE BUILDINGS, 2015, Guimarães. Proceeding ..., Portugal: ANTAC, jul. 2015. v. 2, p. 1501-1511. Disponível em: http://civil.uminho.pt/Euro-ELECS-2015/files/Euro-ELECS_2015-Proceedings_Vol2.pdf. Acesso em: out. 2016.

SHERMAN, W.R.; CRAIG, A.B. Understanding Virtual Reality: interface, application, and design. [sl]: Elsevier, 2003.582 p. Disponível em: http://www.sciencedirect.com/science/book/9781558603530. Acesso em: out. 2016.

SGUIZZARDI, Silvio. Modelando o Futuro: A Evolução do Uso de Tecnologias Digitais no Desenvolvimento de Projetos de Arquitetura. 2011. f 175. Dissertação (Mestrado na área de concentração de Tecnologia da Arquitetura) - Faculdade de Arquitetura e Urbanismo, Universidade de São Paulo, São Paulo, 2011. doi:https://doi.org/10.11606/D.16.2011.tde-26012012153604

VOLK, R.; STENGEL, J.; SCHULTMANN, F.. Building Information Models (BIM) for existing buildings - literature review and future needs. Automation in Construction, v. 38, p.109-127, mar. 2014. ISSN 0926-580. doi:https://doi.org/10.1016/j.autcon.2013.10.023.

WORLD ECONOMIC FORUM, The future of jobs: employment, skills and workforce strategy for the Fourth Industrial Revolution. Janeiro, 2016. Disponível em: https://www.weforum.org/reports/the-future-of-jobs. Acesso em: 05 abril 2017.

\section{${ }^{1}$ Cristiane Lopes Canuto}

Arquiteta. Mestranda do Curso de Mestrado Profissional em Projeto e Patrimônio PROARQ FAU UFRJ. Endereço postal: Rua Barata Ribeiro, 26 apto 1308, Copacabana, Rio de Janeiro - RJ, Brasil, CEP 22011-002

\section{${ }^{2}$ Larissa Moura Ribeiro}

Arquiteta. Mestranda do Curso de Mestrado Profissional em Projeto e Patrimônio PROARQ FAU UFRJ. Endereço postal: Rua Redentor, 102, Paineiras, Juiz de Fora - MG, Brasil, CEP 36016-070

\section{${ }^{3}$ Mônica Santos Salgado}

Professor Titular PROARQ FAU UFRJ, Doutora em Engenharia de Produção COPPE UFRJ Endereço postal: Av. Vice Presidente José Alencar 1400 bloco 1 apto 304, Rio de Janeiro- RJ, Brasil, CEP 22775-033 